\title{
Trudinger's inequality and continuity for Riesz potentials of functions in grand Musielak-Orlicz-Morrey spaces over nondoubling metric measure spaces
}

\author{
Takao Ohno and Tetsu Shimomura
}

\begin{abstract}
In this article we are concerned with Trudinger's inequality and continuity for Riesz potentials of functions in grand Musielak-Orlicz-Morrey spaces over nondoubling metric measure spaces.
\end{abstract}

\section{Introduction}

Grand Lebesgue spaces were introduced in [9] for the study of the Jacobian. They play important roles also in the theory of partial differential equations (see [5], [10], [28], etc.). The generalized grand Lebesgue spaces appeared in [7], where the existence and uniqueness of the nonhomogeneous $N$-harmonic equations were studied.

For $0<\alpha<N$, we define the Riesz potential of order $\alpha$ for a locally integrable function $f$ on $\mathbf{R}^{N}$ by

$$
I_{\alpha} f(x)=\int_{\mathbf{R}^{N}}|x-y|^{\alpha-N} f(y) d y .
$$

The classical Trudinger's inequality for Riesz potentials of $L^{p}$-functions (see, e.g., [2, Theorem 3.1.4(c)]) has been also extended to various function spaces; see [19] and [20] for Morrey spaces of variable exponent, [6] for grand Morrey spaces of variable exponent, [24] for Musielak-Orlicz spaces, and [14] for Musielak-OrliczMorrey spaces. See also [26] and [27]. Recently, Trudinger's inequality has been extended to an inequality for Riesz potentials of functions in grand MusielakOrlicz-Morrey spaces (see [15]).

We denote by $(X, d, \mu)$ a metric measure space, where $X$ is a bounded set, $d$ is a metric on $X$, and $\mu$ is a nonnegative complete Borel regular outer measure on $X$ which is finite in every bounded set. For simplicity, we often write $X$ instead of $(X, d, \mu)$. For $x \in X$ and $r>0$, we denote by $B(x, r)$ the open ball centered at

Kyoto Journal of Mathematics, Vol. 56, No. 3 (2016), 633-653

DOI 10.1215/21562261-3600211, C 2016 by Kyoto University

Received March 2, 2015. Revised June 10, 2015. Accepted June 11, 2015.

2010 Mathematics Subject Classification: Primary 46E35; Secondary 46E30. 
$x$ with radius $r$ and $d_{X}=\sup \{d(x, y): x, y \in X\}$. We assume that $0<d_{X}<\infty$,

$$
\mu(\{x\})=0
$$

for $x \in X$, and $\mu(B(x, r))>0$ for $x \in X$ and $r>0$ for simplicity. In the present article, we do not postulate on $\mu$, the so-called doubling condition. Recall that a Radon measure $\mu$ is said to be doubling if there exists a constant $C>0$ such that $\mu(B(x, 2 r)) \leq C \mu(B(x, r))$ for all $x \in \operatorname{supp}(\mu)(=X)$ and $r>0$. Otherwise $\mu$ is said to be nondoubling.

For $\alpha>0$ and $\tau>0$, we define the Riesz potential of order $\alpha$ for a locally integrable function $f$ on $X$ by (e.g., see [8] and [22])

$$
I_{\alpha, \tau} f(x)=\int_{X} \frac{d(x, y)^{\alpha} f(y)}{\mu(B(x, \tau d(x, y)))} d \mu(y) .
$$

Observe that this naturally extends the Riesz potential operator $I_{\alpha} f(x)$ when $(X, d)$ is the $N$-dimensional Euclidean space and $\mu=d x$.

Our first aim in this article is to give a general version of Trudinger-type exponential integrability for Riesz potentials $I_{\alpha, \tau} f$ of functions in grand MusielakOrlicz-Morrey spaces $\widetilde{L}_{\eta, \xi}^{\Phi, \kappa}(X)$ over nondoubling metric measure spaces $X$ (see, e.g., Corollary 5.5) as an extension of [15, Corollary 6.12] (see Sections 2 and 3 for the definitions of $\Phi, \kappa, \eta, \xi$, and $\left.\widetilde{L}_{\eta, \xi}^{\Phi, \kappa}(X)\right)$. Since we discuss the Morrey version, our strategy is to find estimates of Riesz potentials $I_{\alpha, \tau} f$ by the use of other Riesz-type potentials $I_{\gamma, \tau} f$ of order $\gamma(<\alpha)$, which play the role of the maximal functions (see Section 4). What is new about this article is that we can pass our results to the nondoubling metric measure setting; the technique developed in [14] still works.

On the other hand, beginning with Sobolev's embedding theorem (see, e.g., [1], [2]), continuity properties of Riesz potentials or Sobolev functions have been studied by many authors. See [17] and [18] for generalized Morrey spaces $L^{1, \varphi}$, [21] for Orlicz-Morrey spaces, [21] for variable exponent Morrey spaces, and [19] for two variable exponent Morrey spaces.

Our second aim in this article is to give a general version of continuity for Riesz potentials $I_{\alpha, \tau} f$ of functions in grand Musielak-Orlicz-Morrey spaces over nondoubling metric measure spaces (see, e.g., Corollary 6.6), whose counterpart in the Euclidean setting was not considered in [15]. The result is new even for the Euclidean case.

\section{Preliminaries}

Throughout this article, let $C$ denote various constants independent of the variables in question. In this article, we assume that $X$ is a bounded set, that is, $d_{X}<\infty$. This implies that $\mu(X)<\infty$.

We consider a function

$$
\Phi(x, t)=t \phi(x, t): X \times[0, \infty) \rightarrow[0, \infty)
$$

satisfying the following conditions $(\Phi 1)-(\Phi 4)$ : 
$(\Phi 1) \phi(\cdot, t)$ is measurable on $X$ for each $t \geq 0$ and $\phi(x, \cdot)$ is continuous on $[0, \infty)$ for each $x \in X$;

(Ф2) there exists a constant $A_{1} \geq 1$ such that

$$
A_{1}^{-1} \leq \phi(x, 1) \leq A_{1} \quad \text { for all } x \in X ;
$$

(Ф3) there exists a constant $\varepsilon_{0}>0$ such that $t \mapsto t^{-\varepsilon_{0}} \phi(x, t)$ is uniformly almost increasing, namely, there exists a constant $A_{2} \geq 1$ such that

$$
t^{-\varepsilon_{0}} \phi(x, t) \leq A_{2} s^{-\varepsilon_{0}} \phi(x, s)
$$

for all $x \in X$ whenever $0<t<s$;

(Ф4) there exists a constant $A_{3} \geq 1$ such that

$$
\phi(x, 2 t) \leq A_{3} \phi(x, t) \text { for all } x \in X \text { and } t>0 .
$$

Note that (\$3) implies that

$$
t^{-\varepsilon} \phi(x, t) \leq A_{2} s^{-\varepsilon} \phi(x, s)
$$

for all $x \in X$ and $0<\varepsilon \leq \varepsilon_{0}$ whenever $0<t<s$.

Also note that $(\Phi 2),(\Phi 3)$, and ( $\Phi 4)$ imply

$$
0<\inf _{x \in X} \phi(x, t) \leq \sup _{x \in X} \phi(x, t)<\infty
$$

for each $t>0$ and there exists $\omega>1$ such that

$$
\left(A_{1} A_{2}\right)^{-1} t^{1+\varepsilon_{0}} \leq \Phi(x, t) \leq A_{1} A_{2} A_{3} t^{\omega}
$$

for $t \geq 1$. In fact, we can take $\omega \geq 1+\log A_{3} / \log 2$.

We shall also consider the following condition:

$(\Phi 5)$ for every $\gamma_{1}, \gamma_{2}>0$, there exists a constant $B_{\gamma_{1}, \gamma_{2}} \geq 1$ such that

$$
\phi(x, t) \leq B_{\gamma_{1}, \gamma_{2}} \phi(y, t)
$$

whenever $d(x, y) \leq \gamma_{1} t^{-1 / \gamma_{2}}$ and $t \geq 1$.

Let $\bar{\phi}(x, t)=\sup _{0 \leq s \leq t} \phi(x, s)$, and let

$$
\bar{\Phi}(x, t)=\int_{0}^{t} \bar{\phi}(x, r) d r
$$

for $x \in X$ and $t \geq 0$. Then $\bar{\Phi}(x, \cdot)$ is convex and

$$
\frac{1}{2 A_{3}} \Phi(x, t) \leq \bar{\Phi}(x, t) \leq A_{2} \Phi(x, t)
$$

for all $x \in X$ and $t \geq 0$.

EXAMPLE 2.1

Let $p(\cdot)$ and $q_{j}(\cdot), j=1, \ldots, k$, be measurable functions on $X$ such that

$$
1<p^{-}:=\inf _{x \in X} p(x) \leq \sup _{x \in X} p(x)=: p^{+}<\infty
$$

and 


$$
-\infty<q_{j}^{-}:=\inf _{x \in X} q_{j}(x) \leq \sup _{x \in X} q_{j}(x)=: q_{j}^{+}<\infty, \quad j=1, \ldots, k .
$$

Set $L(t):=\log (e+t)$, set $L^{(1)}(t)=L(t)$, and set $L^{(j)}(t)=L\left(L^{(j-1)}(t)\right), j=2, \ldots$. Then,

$$
\Phi_{p(\cdot),\left\{q_{j}(\cdot)\right\}}(x, t)=t^{p(x)} \prod_{j=1}^{k}\left(L^{(j)}(t)\right)^{q_{j}(x)}
$$

satisfies $(\Phi 1),(\Phi 2),(\Phi 3)$ with $0<\varepsilon_{0}<p^{-}-1$, and $(\Phi 4)$. For any $\omega>p^{+}$, (2.1) holds. Then, $\Phi_{p(\cdot),\left\{q_{j}(\cdot)\right\}}(x, t)$ satisfies $(\Phi 5)$ if $p(\cdot)$ is log-Hölder continuous, namely,

$$
|p(x)-p(y)| \leq \frac{C_{p}}{L(1 / d(x, y))} \quad(x \neq y),
$$

and $q_{j}(\cdot)$ is $(j+1)$-log-Hölder continuous, namely,

$$
\left|q_{j}(x)-q_{j}(y)\right| \leq \frac{C_{q_{j}}}{L^{(j+1)}(1 / d(x, y))} \quad(x \neq y)
$$

for $j=1, \ldots, k$ (cf. [13, Example 2.1]).

We also consider a function $\kappa(x, r): X \times\left(0, d_{X}\right) \rightarrow(0, \infty)$ satisfying the following conditions:

$(\kappa 1) \kappa(x, \cdot)$ is continuous on $\left(0, d_{X}\right)$ for each $x \in X$ and satisfies the uniform doubling condition: there is a constant $Q_{1} \geq 1$ such that

$$
Q_{1}^{-1} \kappa(x, r) \leq \kappa\left(x, r^{\prime}\right) \leq Q_{1} \kappa(x, r)
$$

for all $x \in X$ whenever $0<r \leq r^{\prime} \leq 2 r<d_{X}$;

( $\kappa 2) r \mapsto r^{-\delta} \kappa(x, r)$ is uniformly almost increasing for some $\delta>0$, namely, there is a constant $Q_{2}>0$ such that

$$
r^{-\delta} \kappa(x, r) \leq Q_{2} s^{-\delta} \kappa(x, s)
$$

for all $x \in X$ whenever $0<r<s<d_{X}$;

$(\kappa 3)$ there are constants $Q>0$ and $Q_{3} \geq 1$ such that

$$
Q_{3}^{-1} \min \left(1, r^{Q}\right) \leq \kappa(x, r) \leq Q_{3}
$$

for all $x \in X$ and $0<r<d_{X}$.

\section{EXAMPLE 2.2}

Let $\nu(\cdot)$ and $\beta(\cdot)$ be functions on $X$ such that $\nu^{-}:=\inf _{x \in X} \nu(x)>0, \nu^{+}:=$ $\sup _{x \in X} \nu(x) \leq Q$, and $-c(Q-\nu(x)) \leq \beta(x) \leq c$ for all $x \in X$ and some constant $c>0$. Then $\kappa(x, r)=r^{\nu(x)}(\log (e+1 / r))^{\beta(x)}$ satisfies $(\kappa 1),(\kappa 2)$, and $(\kappa 3)$; we can take any $0<\delta<\nu^{-}$for $(\kappa 2)$.

We say that $f$ is a locally integrable function on $X$ if $f$ is an integrable function on all balls $B$ in $X$. Given $\Phi(x, t)$ satisfying $(\Phi 1),(\Phi 2),(\Phi 3)$, and $(\Phi 4)$ and 
$\kappa(x, r)$ satisfying $(\kappa 1),(\kappa 2)$, and $(\kappa 3)$, we define the Musielak-Orlicz-Morrey space $L^{\Phi, \kappa}(X)$ by

$$
\begin{aligned}
& L^{\Phi, \kappa}(X) \\
& \quad=\left\{f \in L_{\mathrm{loc}}^{1}(X) ; \sup _{x \in X, 0<r<d_{X}} \frac{\kappa(x, r)}{\mu(B(x, r))} \int_{B(x, r) \cap X} \Phi(y,|f(y)|) d \mu(y)<\infty\right\} .
\end{aligned}
$$

It is a Banach space with respect to the norm (cf. [23])

$$
\begin{aligned}
& \|f\|_{\Phi, \kappa ; X} \\
& \quad=\inf \left\{\lambda>0 ; \sup _{x \in X, 0<r<d_{X}} \frac{\kappa(x, r)}{\mu(B(x, r))} \int_{B(x, r) \cap X} \bar{\Phi}(y,|f(y)| / \lambda) d \mu(y) \leq 1\right\} .
\end{aligned}
$$

\section{Grand Musielak-Orlicz-Morrey space}

For $\varepsilon \geq 0$, set $\Phi_{\varepsilon}(x, t):=t^{-\varepsilon} \Phi(x, t)=t^{1-\varepsilon} \phi(x, t)$. Then, $\Phi_{\varepsilon}(x, t)$ satisfies $(\Phi 1)$,

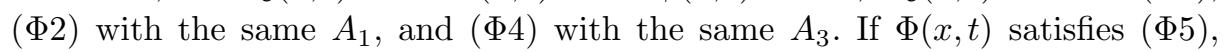
then so does $\Phi_{\varepsilon}(x, t)$ with the same $\left\{B_{\gamma_{1}, \gamma_{2}}\right\}_{\gamma_{1}, \gamma_{2}>0}$.

If $0 \leq \varepsilon<\varepsilon_{0}$, then $\Phi_{\varepsilon}(x, t)$ satisfies ( $\left.\Phi 3\right)$ with $\varepsilon_{0}$ replaced by $\varepsilon_{0}-\varepsilon$ and the same $A_{2}$. It follows that

$$
\frac{1}{2 A_{3}} \Phi_{\varepsilon}(x, t) \leq \overline{\Phi_{\varepsilon}}(x, t) \leq A_{2} \Phi_{\varepsilon}(x, t)
$$

for all $x \in X, t \geq 0$, and $0 \leq \varepsilon \leq \varepsilon_{0}$.

Let

$$
\tilde{\sigma}=\sup \left\{\sigma \geq 0: r^{Q-\sigma} \kappa(x, r)^{-1} \text { is bounded on } X \times\left(0, \min \left(1, d_{X}\right)\right)\right\} .
$$

By $(\kappa 2)$ and $(\kappa 3), 0 \leq \tilde{\sigma} \leq Q$. If $\tilde{\sigma}=0$, then let $\sigma_{0}=0$; otherwise fix any $\sigma_{0} \in$ $(0, \tilde{\sigma})$. We also take $\delta_{0}$ such that $0<\delta_{0}<\delta$ for $\delta$ in $(\kappa 2)$.

For $-\delta_{0} \leq \sigma \leq \sigma_{0}$, set

$$
\kappa_{\sigma}(x, r)=r^{\sigma} \kappa(x, r)
$$

for $x \in X$ and $0<r<d_{X}$. Then $\kappa_{\sigma}(x, r)$ satisfies $(\kappa 1),(\kappa 2)$, and $(\kappa 3)$ with constants independent of $\sigma$.

\section{LEMMA 3.1 ([15, PROPOSITION 3.2])}

Assume that $\Phi(x, t)$ satisfies ( $\Phi 5)$. If $0 \leq \varepsilon_{1} \leq \varepsilon_{2} \leq \varepsilon_{0},-\delta_{0} \leq \sigma_{j} \leq \sigma_{0}, j=1,2$, and

$$
\sigma_{1}+\frac{\delta-\delta_{0}}{\omega} \varepsilon_{1} \leq \sigma_{2}+\frac{\delta-\delta_{0}}{\omega} \varepsilon_{2}
$$

then $L^{\Phi_{\varepsilon_{1}}, \kappa_{\sigma_{1}}}(X) \subset L^{\Phi_{\varepsilon_{2}}, \kappa_{\sigma_{2}}}(X)$ and

$$
\|f\|_{\Phi_{\varepsilon_{2}}, \kappa_{\sigma_{2}} ; X} \leq C\|f\|_{\Phi_{\varepsilon_{1}}, \kappa_{\sigma_{1}} ; X}
$$

for all $f \in L^{\Phi_{\varepsilon_{1}}, \kappa_{\sigma_{1}}}(X)$ with $C>0$ independent of $\varepsilon_{1}, \varepsilon_{2}, \sigma_{1}, \sigma_{2}$. 
In particular,

$$
L^{\Phi, \kappa}(X) \subset L^{\Phi_{\varepsilon}, \kappa_{\sigma}}(X)
$$

if $0 \leq \varepsilon \leq \varepsilon_{0},-\delta_{0} \leq \sigma \leq \sigma_{0}$, and $\sigma+\left(\left(\delta-\delta_{0}\right) / \omega\right) \varepsilon \geq 0$.

Let $\eta(\varepsilon)$ be an increasing positive function on $(0, \infty)$ such that $\eta(0+)=0$. Let $\xi(\varepsilon)$ be a function on $\left(0, \varepsilon_{1}\right]$ with some $\varepsilon_{1} \in\left(0, \varepsilon_{0} / 2\right]$ such that $-\delta_{0} \leq \xi(\varepsilon) \leq \sigma_{0}$ for $0<\varepsilon \leq \varepsilon_{1}, \xi(0+)=0$, and $\varepsilon \mapsto \xi(\varepsilon)+\left(\left(\delta-\delta_{0}\right) / \omega\right) \varepsilon$ is nondecreasing; in particular, $\xi(\varepsilon)+\left(\left(\delta-\delta_{0}\right) / \omega\right) \varepsilon \geq 0$ for $0<\varepsilon \leq \varepsilon_{1}$.

Given $\Phi(x, t), \kappa(x, r), \eta(\varepsilon)$, and $\xi(\varepsilon)$, the associated (generalized) grand Musielak-Orlicz-Morrey space is defined by (cf. [11] for generalized grand Morrey space)

$$
\widetilde{L}_{\eta, \xi}^{\Phi, \kappa}(X)=\left\{f \in \bigcap_{0<\varepsilon \leq \varepsilon_{1}} L^{\Phi_{\varepsilon}, \kappa_{\xi(\varepsilon)}}(X) ;\|f\|_{\Phi, \kappa ; \eta, \xi ; X}<\infty\right\}
$$

where

$$
\|f\|_{\Phi, \kappa ; \eta, \xi ; X}=\sup _{0<\varepsilon \leq \varepsilon_{1}} \eta(\varepsilon)\|f\|_{\Phi_{\varepsilon}, \kappa_{\xi(\varepsilon)} ; X} .
$$

Note that $\widetilde{L}_{\eta, \xi}^{\Phi, \kappa}(X)$ is a Banach space with the norm $\|f\|_{\Phi, \kappa ; \eta, \xi ; X}$. Also note that, in view of Lemma 3.1, this space is determined independent of the choice of $\varepsilon_{1}$.

\section{REMARK 3.2}

If $\mu(B(x, r))$ satisfies $(\kappa 1),(\kappa 2)$, and $(\kappa 3)$, then the associated (generalized) grand Musielak-Orlicz-Morrey space $\widetilde{L}_{\eta, \xi}^{\Phi, \kappa}(X)$ includes the following spaces:

- generalized grand Lebesgue spaces introduced in [3] if $\kappa(x, r)=\mu(B(x, r))$ and $\xi(\varepsilon) \equiv 0$

- grand Orlicz spaces introduced in [12] (see also [4]) if $\kappa(x, r)=\mu(B(x, r)$ ), $\xi(\varepsilon) \equiv 0, \Phi(x, t)=\Phi(t)$, and

$$
\sup _{0<\varepsilon \leq \varepsilon_{0}} \eta(\varepsilon) \int_{1}^{\infty} t^{-N-\varepsilon} \Phi(t) \frac{d t}{t}<\infty ;
$$

- grand Morrey spaces introduced in [16] if $\xi(\varepsilon) \equiv 0$;

- grand grand Morrey spaces introduced in [25] and generalized grand Morrey spaces introduced in [11] if $\xi(\varepsilon)$ is an increasing positive function on $(0, \infty)$.

\section{Lemmas}

\section{LEMMA 4.1 ([13, LEMMA 5.1])}

Let $F(x, t)$ be a positive function on $X \times(0, \infty)$ satisfying the following conditions:

(F1) $F(x, \cdot)$ is continuous on $(0, \infty)$ for each $x \in X$;

(F2) there exists a constant $K_{1} \geq 1$ such that

$$
K_{1}^{-1} \leq F(x, 1) \leq K_{1} \quad \text { for all } x \in X ;
$$


(F3) $t \mapsto t^{-\varepsilon} F(x, t)$ is uniformly almost increasing for some $\varepsilon>0$; namely, there exists a constant $K_{2} \geq 1$ such that

$$
t^{-\varepsilon} F(x, t) \leq K_{2} s^{-\varepsilon} F(x, s) \quad \text { for all } x \in X \text { whenever } 0<t<s .
$$

Set

$$
F^{-1}(x, s)=\sup \{t>0 ; F(x, t)<s\}
$$

for $x \in X$ and $s>0$. Then:

(1) $F^{-1}(x, \cdot)$ is nondecreasing.

$$
F^{-1}(x, \lambda t) \leq\left(K_{2} \lambda\right)^{1 / \varepsilon} F^{-1}(x, t)
$$

for all $x \in X, t>0$, and $\lambda \geq 1$.

$$
F\left(x, F^{-1}(x, t)\right)=t
$$

for all $x \in X$ and $t>0$.

(4)

$$
K_{2}^{-1 / \varepsilon} t \leq F^{-1}(x, F(x, t)) \leq K_{2}^{2 / \varepsilon} t
$$

for all $x \in X$ and $t>0$.

$$
\min \left\{1,\left(\frac{s}{K_{1} K_{2}}\right)^{1 / \varepsilon}\right\} \leq F^{-1}(x, s) \leq \max \left\{1,\left(K_{1} K_{2} s\right)^{1 / \varepsilon}\right\}
$$

for all $x \in X$ and $s>0$.

\section{REMARK 4.2}

We have that $F(x, t)=\Phi(x, t)$ satisfies (F1), (F2), and (F3) with $K_{1}=A_{1}, K_{2}=$ $A_{2}$, and $\varepsilon=1$.

By $(\kappa 3)$ and (4.3), we have the following result.

\section{LEMMA 4.3}

There exists a constant $C>0$ such that

$$
C^{-1} \leq \Phi^{-1}\left(x, \kappa(x, r)^{-1}\right) \leq C r^{-Q}
$$

for all $x \in X$ and $0<r \leq d_{X}$, where $Q$ is a constant appearing in $(\kappa 3)$.

LEMMA 4.4 (CF. [15, LEMMA 3.1])

There exist constants $C \geq 1$ and $r_{0} \in\left(0, \min \left(1, d_{X}\right)\right)$ such that $\kappa_{\sigma}(x, r) \leq C r^{\delta-\delta_{0}}$ and

$$
C^{-1} r^{-\left(\delta-\delta_{0}\right) / \omega} \leq \Phi_{\varepsilon}^{-1}\left(x, \kappa_{\sigma}(x, r)^{-1}\right) \leq C r^{-Q}
$$

for all $x \in X, 0<r \leq r_{0},-\delta_{0} \leq \sigma \leq \sigma_{0}$, and $0<\varepsilon \leq \varepsilon_{0}$, where $Q$ is a constant appearing in $(\kappa 3)$. 


\section{Proof}

In view of the proof of [15, Lemma 3.1], we only have to prove that there exists a constant $C \geq 1$ such that

$$
\Phi_{\varepsilon}^{-1}\left(x, \kappa_{\sigma}(x, r)^{-1}\right) \leq C r^{-Q}
$$

for all $x \in X, 0<r \leq r_{0},-\delta_{0} \leq \sigma \leq \sigma_{0}$, and $0<\varepsilon \leq \varepsilon_{0}$. First note from ( $\left.\Phi 3\right)$ that there exists a constant $C \geq 1$ such that

$$
t^{-\varepsilon^{\prime}} \Phi_{\varepsilon}(x, t) \leq C s^{-\varepsilon^{\prime}} \Phi_{\varepsilon}(x, s)
$$

for all $x \in X$ and $0<\varepsilon^{\prime} \leq \varepsilon_{0}-\varepsilon+1$ whenever $0<t<s$. By Lemma 4.1(5) with $\varepsilon^{\prime}=1$ and $(\kappa 3)$, we have

$$
\Phi_{\varepsilon}^{-1}\left(x, \kappa_{\sigma}(x, r)^{-1}\right) \leq C \kappa_{\sigma}(x, r)^{-1} \leq C r^{-Q}
$$

for all $x \in X, 0<r \leq r_{0},-\delta_{0} \leq \sigma \leq \sigma_{0}$, and $0<\varepsilon \leq \varepsilon_{0}$, as required.

From now on, we assume:

$(\Xi) \xi(\varepsilon) \leq a \varepsilon$ for $0<\varepsilon \leq \varepsilon_{1}$ with some $a \geq 0$.

Recall that $\xi(\varepsilon) \geq-\left(\left(\delta-\delta_{0}\right) / \omega\right) \varepsilon$ by assumption. Let

$$
\varepsilon(r)=(\log (e+1 / r))^{-1}
$$

for $r>0$, and let $r_{1} \in\left(0, \min \left(1, d_{X}\right)\right)$ be such that $\varepsilon(r) \leq \varepsilon_{1}$ for $0<r \leq r_{1}$.

\section{LEMMA 4.5 ([15, LEMMA 6.2])}

There exists a constant $C \geq 1$ such that

$$
C^{-1} \Phi^{-1}\left(x, \kappa(x, r)^{-1}\right) \leq \Phi_{\varepsilon(r)}^{-1}\left(x, \kappa_{\xi(\varepsilon(r))}(x, r)^{-1}\right) \leq C \Phi^{-1}\left(x, \kappa(x, r)^{-1}\right)
$$

for all $x \in X$ and $0<r \leq r_{1}$.

\section{LEMMA 4.6}

Assume that $\Phi(x, t)$ satisfies $(\Phi 5)$. Then there exists a constant $C>0$ such that

$$
\frac{1}{\mu(B(x, r))} \int_{B(x, r) \cap X} f(y) d \mu(y) \leq C \Phi^{-1}\left(x, \kappa(x, r)^{-1}\right) \eta\left((\log (e+1 / r))^{-1}\right)^{-1}
$$

for all $x \in X, 0<r<d_{X}$, and nonnegative $f \in \widetilde{L}_{\eta, \xi}^{\Phi, \kappa}(X)$ with $\|f\|_{\Phi, \kappa ; \eta, \xi ; X} \leq 1$.

Proof

Let $f$ be a nonnegative function with $\|f\|_{\Phi, \kappa ; \eta, \xi ; X} \leq 1$. Then note from (3.1) that

$$
\frac{\kappa_{\xi(\varepsilon)}(x, r)}{\mu(B(x, r))} \int_{B(x, r) \cap X} \Phi_{\varepsilon}(y, \eta(\varepsilon) f(y)) d \mu(y) \leq 2 A_{3}
$$

for $x \in X, 0<r<d_{X}$, and $0<\varepsilon<\varepsilon_{1}$, so that

$$
\frac{\kappa_{\xi(\varepsilon(r))}(x, r)}{\mu(B(x, r))} \int_{B(x, r) \cap X} \Phi_{\varepsilon(r)}(y, \eta(\varepsilon(r)) f(y)) d \mu(y) \leq 2 A_{3}
$$


for $x \in X$ and $0<r \leq r_{1}$. Let $g_{r}(y)=\eta(\varepsilon(r)) f(y)$, and let

$$
K(x, r)=\Phi_{\varepsilon(r)}^{-1}\left(x, \kappa_{\xi(\varepsilon(r))}(x, r)^{-1}\right) .
$$

Since there exist constants $C \geq 1$ and $r_{0} \in\left(0, \min \left(1, d_{X}\right)\right)$ such that

$$
1 \leq K(x, r) \leq C r^{-Q}
$$

for all $x \in X$ and $0<r \leq \min \left\{r_{0}, r_{1}\right\}$ by Lemma 4.4, we see from ( $\left.\Phi 5\right)$ and (4.2) that

$$
\Phi_{\varepsilon(r)}(y, K(x, r)) \geq C \Phi_{\varepsilon(r)}(x, K(x, r))=C \kappa_{\xi(\varepsilon(r))}(x, r)^{-1}
$$

for all $y \in B(x, r)$ and $0<r \leq \min \left\{r_{0}, r_{1}\right\}$. Therefore, we have by $(\Phi 3)$

$$
\begin{aligned}
& \frac{1}{\mu(B(x, r))} \int_{B(x, r) \cap X} g_{r}(y) d \mu(y) \\
& \quad \leq K(x, r)+\frac{A_{2}}{\mu(B(x, r))} \int_{B(x, r) \cap X} g_{r}(y) \frac{g_{r}(y)^{-1} \Phi_{\varepsilon(r)}\left(y, g_{r}(y)\right)}{K(x, r)^{-1} \Phi_{\varepsilon(r)}(y, K(x, r))} d \mu(y) \\
& \quad \leq C K(x, r)\left\{1+\frac{\kappa_{\xi(\varepsilon(r))}(x, r)}{\mu(B(x, r))} \int_{B(x, r) \cap X} \Phi_{\varepsilon(r)}\left(y, g_{r}(y)\right) d \mu(y)\right\} \\
& \quad \leq C K(x, r)
\end{aligned}
$$

for $x \in X$ and $0<r \leq \min \left\{r_{0}, r_{1}\right\}$. Hence, we find by Lemma 4.5

$$
\frac{1}{\mu(B(x, r))} \int_{B(x, r) \cap X} g_{r}(y) d \mu(y) \leq C \Phi^{-1}\left(x, \kappa(x, r)^{-1}\right)
$$

for all $x \in X$ and $0<r \leq \min \left\{r_{0}, r_{1}\right\}$.

When $\min \left\{r_{0}, r_{1}\right\}<r<d_{X}$, we have by (4.4)

$\frac{1}{\mu(B(x, r))} \int_{B(x, r) \cap X} f(y) d \mu(y) \leq C \leq C \Phi^{-1}\left(x, \kappa(x, r)^{-1}\right) \eta\left((\log (e+1 / r))^{-1}\right)^{-1}$, as required.

Set

$$
\Gamma(x, s)=\int_{1 / s}^{d_{X}} \rho^{\alpha} \Phi^{-1}\left(x, \kappa(x, \rho)^{-1}\right) \eta\left((\log (e+1 / \rho))^{-1}\right)^{-1} \frac{d \rho}{\rho}
$$

for $s \geq 2 / d_{X}$ and $x \in X$. For $0 \leq s<2 / d_{X}$ and $x \in X$, we set $\Gamma(x, s)=$ $\Gamma\left(x, 2 / d_{X}\right)\left(d_{X} / 2\right) s$. Then note that $\Gamma(x, \cdot)$ is strictly increasing and continuous for each $x \in X$.

LEMMA 4.7 (CF. [14, LEMMA 3.5])

There exists a positive constant $C^{\prime}$ such that $\Gamma\left(x, 2 / d_{X}\right) \geq C^{\prime}$ for all $x \in X$.

\section{LEMMA 4.8}

Assume that $\Phi(x, t)$ satisfies $(\Phi 5)$. Let $\tau>1$. Then there exists a constant $C>0$ such that 


$$
\int_{X \backslash B(x, \delta)} \frac{d(x, y)^{\alpha} f(y)}{\mu(B(x, \tau d(x, y)))} d \mu(y) \leq C \Gamma\left(x, \frac{1}{\delta}\right)
$$

for all $x \in X, 0<\delta \leq d_{X} / 2$, and nonnegative $f \in \widetilde{L}_{\eta, \xi}^{\Phi, \kappa}(X)$ with $\|f\|_{\Phi, \kappa ; \eta, \xi ; X} \leq 1$.

Proof

Let $j_{0}$ be the smallest positive integer such that $\tau^{j_{0}} \delta \geq d_{X}$. By Lemma 4.6, we have

$$
\begin{aligned}
& \int_{X \backslash B(x, \delta)} \frac{d(x, y)^{\alpha} f(y)}{\mu(B(x, \tau d(x, y)))} d \mu(y) \\
& =\sum_{j=1}^{j_{0}} \int_{X \cap\left(B\left(x, \tau^{j} \delta\right) \backslash B\left(x, \tau^{j-1} \delta\right)\right)} \frac{d(x, y)^{\alpha} f(y)}{\mu(B(x, \tau d(x, y)))} d \mu(y) \\
& \leq \sum_{j=1}^{j_{0}}\left(\tau^{j} \delta\right)^{\alpha} \frac{1}{\mu\left(B\left(x, \tau^{j} \delta\right)\right)} \int_{X \cap B\left(x, \tau^{j} \delta\right)} f(y) d \mu(y) \\
& \leq C\left(\sum_{j=1}^{j_{0}-1}\left(\tau^{j} \delta\right)^{\alpha} \Phi^{-1}\left(x, \kappa\left(x, \tau^{j} \delta\right)^{-1}\right) \eta\left(\left(\log \left(e+1 /\left(\tau^{j} \delta\right)\right)\right)^{-1}\right)^{-1}\right. \\
& \left.\quad+d_{X}^{\alpha} \Phi^{-1}\left(x, \kappa\left(x, d_{X}\right)^{-1}\right) \eta\left(\left(\log \left(e+1 / d_{X}\right)\right)^{-1}\right)^{-1}\right)
\end{aligned}
$$

where we assume that $\sum_{j=1}^{0} a_{j}=0$ for $a_{j} \in \mathbf{R}$. By $(\kappa 2)$ and (4.1), we have

$$
\begin{aligned}
& \int_{\tau^{j-1} \delta}^{\tau^{j} \delta} t^{\alpha} \Phi^{-1}\left(x, \kappa(x, t)^{-1}\right) \eta\left((\log (e+1 / t))^{-1}\right)^{-1} \frac{d t}{t} \\
& \quad \geq\left(\tau^{j-1} \delta\right)^{\alpha} \Phi^{-1}\left(x, Q_{2}^{-1} \kappa\left(x, \tau^{j} \delta\right)^{-1}\right) \eta\left(\left(\log \left(e+1 /\left(\tau^{j} \delta\right)\right)\right)^{-1}\right)^{-1} \log \tau \\
& \quad \geq \frac{\left(\tau^{j} \delta\right)^{\alpha} \log \tau}{\tau^{\alpha} A_{2} Q_{2}} \Phi^{-1}\left(x, \kappa\left(x, \tau^{j} \delta\right)^{-1}\right) \eta\left(\left(\log \left(e+1 /\left(\tau^{j} \delta\right)\right)\right)^{-1}\right)^{-1} \\
& \quad=C\left(\tau^{j} \delta\right)^{\alpha} \log \tau \Phi^{-1}\left(x, \kappa\left(x, \tau^{j} \delta\right)^{-1}\right) \eta\left(\left(\log \left(e+1 /\left(\tau^{j} \delta\right)\right)\right)^{-1}\right)^{-1}
\end{aligned}
$$

and

$$
\begin{aligned}
& \int_{d_{X} / 2}^{d_{X}} t^{\alpha} \Phi^{-1}\left(x, \kappa(x, t)^{-1}\right) \eta\left((\log (e+1 / t))^{-1}\right)^{-1} \frac{d t}{t} \\
& \geq \frac{d_{X}^{\alpha} \log 2}{2^{\alpha} A_{2} Q_{2}} \Phi^{-1}\left(x, \kappa\left(x, d_{X}\right)^{-1}\right) \eta\left(\left(\log \left(e+1 / d_{X}\right)\right)^{-1}\right)^{-1} \\
& =C d_{X}^{\alpha} \Phi^{-1}\left(x, \kappa\left(x, d_{X}\right)^{-1}\right) \eta\left(\left(\log \left(e+1 / d_{X}\right)\right)^{-1}\right)^{-1}
\end{aligned}
$$

Hence, we obtain

$$
\begin{aligned}
& \int_{X \backslash B(x, \delta)} \frac{d(x, y)^{\alpha} f(y)}{\mu(B(x, \tau d(x, y)))} d \mu(y) \\
& \leq \frac{C}{\log \tau}\left(\sum_{j=1}^{j_{0}-1} \int_{\tau^{j-1} \delta}^{\tau^{j} \delta} t^{\alpha} \Phi^{-1}\left(x, \kappa(x, t)^{-1}\right) \eta\left((\log (e+1 / t))^{-1}\right)^{-1} \frac{d t}{t}\right.
\end{aligned}
$$




$$
\begin{aligned}
& \left.+\int_{d_{X} / 2}^{d_{X}} t^{\alpha} \Phi^{-1}\left(x, \kappa(x, t)^{-1}\right) \eta\left((\log (e+1 / t))^{-1}\right)^{-1} \frac{d t}{t}\right) \\
\leq & \frac{C}{\log \tau} \Gamma\left(x, \frac{1}{\delta}\right),
\end{aligned}
$$

as required.

\section{LEMMA 4.9}

Assume that $\Phi(x, t)$ satisfies $(\Phi 5)$. Let $\tau>2$ and $\vartheta>1$ be such that $\tau>(\vartheta+$ 1) $/(\vartheta-1)$. Let $\gamma>0$, and define

$$
\lambda_{\gamma}(z, r)=\frac{1}{1+\int_{r}^{d_{X}} \rho^{\gamma} \Phi^{-1}\left(z, \kappa(z, \rho)^{-1}\right) \eta\left((\log (e+1 / \rho))^{-1}\right)^{-1} \frac{d \rho}{\rho}}
$$

for $z \in X$ and $0<r<d_{X}$. Then there exists a constant $C_{I, \gamma}>0$ such that

$$
\frac{\lambda_{\gamma}(z, r)}{\mu(B(z, \vartheta r))} \int_{X \cap B(z, r)} I_{\gamma, \tau} f(x) d \mu(x) \leq C_{I, \gamma}
$$

for all $z \in X, 0<r<d_{X}$, and nonnegative $f \in \widetilde{L}_{\eta, \xi}^{\Phi, \kappa}(X)$ with $\|f\|_{\Phi, \kappa ; \eta, \xi ; X} \leq 1$.

Proof

Let $z \in X$, and let $0<r<d_{X}$. Write

$$
\begin{aligned}
I_{\gamma, \tau} f(x)= & \int_{X \cap B(z, \vartheta r)} \frac{d(x, y)^{\gamma} f(y)}{\mu(B(x, \tau d(x, y)))} d \mu(y) \\
& +\int_{X \backslash B(z, \vartheta r)} \frac{d(x, y)^{\gamma} f(y)}{\mu(B(x, \tau d(x, y))} d \mu(y) \\
= & I_{1}(x)+I_{2}(x)
\end{aligned}
$$

for $x \in B(z, r)$. By Fubini's theorem,

$$
\begin{aligned}
& \int_{X \cap B(z, r)} I_{1}(x) d \mu(x) \\
& \quad=\int_{X \cap B(z, \vartheta r)}\left(\int_{X \cap B(z, r)} \frac{d(x, y)^{\gamma}}{\mu(B(x, \tau d(x, y)))} d \mu(x)\right) f(y) d \mu(y) \\
& \quad \leq \int_{X \cap B(z, \vartheta r)}\left(\int_{X \cap B(y,(\vartheta+1) r)} \frac{d(x, y)^{\gamma}}{\mu(B(x, \tau d(x, y)))} d \mu(x)\right) f(y) d \mu(y) .
\end{aligned}
$$

Hence,

$$
\begin{aligned}
& \int_{X \cap B(z, r)} I_{1}(x) d \mu(x) \\
& \quad \leq \int_{X \cap B(z, \vartheta r)}\left(\sum_{j=1}^{\infty} \int_{X \cap\left(B\left(y, R_{j}\right) \backslash B\left(y, R_{j+1}\right)\right)} \frac{d(x, y)^{\gamma}}{\mu(B(x, \tau d(x, y)))} d \mu(x)\right) f(y) d \mu(y) \\
& \quad \leq \int_{X \cap B(z, \vartheta r)}\left(\sum_{j=1}^{\infty} \int_{X \cap\left(B\left(y, R_{j}\right) \backslash B\left(y, R_{j+1}\right)\right)} \frac{R_{j}^{\gamma}}{\mu\left(B\left(x, \tau R_{j+1}\right)\right)} d \mu(x)\right) f(y) d \mu(y)
\end{aligned}
$$




$$
\begin{aligned}
& \leq \int_{X \cap B(z, \vartheta r)}\left(\sum_{j=1}^{\infty} \int_{X \cap\left(B\left(y, R_{j}\right) \backslash B\left(y, R_{j+1}\right)\right)} \frac{R_{j}^{\gamma}}{\mu\left(B\left(y, R_{j}\right)\right)} d \mu(x)\right) f(y) d \mu(y) \\
& \leq \int_{X \cap B(z, \vartheta r)}\left(\sum_{j=1}^{\infty} R_{j}^{\gamma}\right) f(y) d \mu(y) \\
& =\frac{(\vartheta+1)^{\gamma}(\tau / 2)^{\gamma}}{(\tau / 2)^{\gamma}-1} r^{\gamma} \int_{X \cap B(z, \vartheta r)} f(y) d \mu(y),
\end{aligned}
$$

where $R_{j}=(\vartheta+1)(\tau / 2)^{-j+1} r$. Now, by Lemma 4.6, $(\kappa 2)$, and (4.1), we have

$$
\begin{aligned}
& r^{\gamma} \int_{X \cap B(z, \vartheta r)} f(y) d \mu(y) \\
& \quad \leq C r^{\gamma} \mu(B(z, \vartheta r)) \Phi^{-1}\left(z, \kappa(z, \vartheta r)^{-1}\right) \eta\left((\log (e+1 /(\vartheta r)))^{-1}\right)^{-1} \\
& \quad \leq \frac{C}{\log \vartheta} \mu(B(z, \vartheta r)) \int_{r}^{\vartheta r} \rho^{\gamma} \Phi^{-1}\left(z, \kappa(z, \rho)^{-1}\right) \eta\left((\log (e+1 / \rho))^{-1}\right)^{-1} \frac{d \rho}{\rho}
\end{aligned}
$$

if $0<r \leq d_{X} / \vartheta$, and by Lemma 4.6 and (4.4), we have

$$
\begin{aligned}
& r^{\gamma} \int_{X \cap B(z, \vartheta r)} f(y) d \mu(y) \\
& \quad=r^{\gamma} \int_{B\left(z, d_{X}\right)} f(y) d \mu(y) \\
& \quad \leq C d_{X}^{\gamma} \mu\left(B\left(z, d_{X}\right)\right) \Phi^{-1}\left(z, \kappa\left(z, d_{X}\right)^{-1}\right) \eta\left(\left(\log \left(e+1 / d_{X}\right)\right)^{-1}\right)^{-1} \\
& \quad \leq C \mu(B(z, \vartheta r))
\end{aligned}
$$

if $d_{X} / \vartheta<r<d_{X}$. Therefore,

$$
\int_{X \cap B(z, r)} I_{1}(x) d \mu(x) \leq \frac{C}{\left((\tau / 2)^{\gamma}-1\right) \log \vartheta} \frac{\mu(B(z, \vartheta r))}{\lambda_{\gamma}(z, r)}
$$

for all $0<r<d_{X}$.

Set $c=(\tau(\vartheta-1)-1) / \vartheta>1$. For $I_{2}$, first note that $I_{2}(x)=0$ if $x \in X$ and $r \geq d_{X} / \vartheta$. Let $0<r<d_{X} / \vartheta$. Let $j_{0}$ be the smallest positive integer such that $\vartheta c^{j_{0}} r \geq d_{X}$. Here we claim that $x \in B(z, r)$ and $y \in X \backslash B(z, \vartheta r)$ imply that

$$
d(y, z) \leq \frac{\vartheta}{\vartheta-1} d(x, y)
$$

and

$$
B(z, c d(z, y)) \subset B(x, \tau d(x, y)) .
$$

Indeed, we have $d(x, z)<r$ and $d(y, z) \geq \vartheta r$. Hence, it follows that

$$
d(y, z) \leq d(x, y)+d(x, z) \leq d(x, y)+\frac{1}{\vartheta} d(y, z),
$$

which yields (4.5). Also observe that, when $w \in B(z, c d(z, y))$, we have by (4.5) 


$$
\begin{aligned}
d(w, x) & \leq d(z, x)+d(w, z) \\
& \leq \frac{1}{\vartheta} d(z, y)+c d(z, y) \\
& \leq\left(c+\frac{1}{\vartheta}\right) \frac{\vartheta}{\vartheta-1} d(x, y)=\tau d(x, y),
\end{aligned}
$$

which yields (4.6).

Consequently it follows from (4.6) that

$$
I_{2}(x) \leq C \int_{X \backslash B(z, \vartheta r)} \frac{d(z, y)^{\gamma} f(y)}{\mu(B(z, c d(z, y)))} d \mu(y) \quad \text { for } x \in X \cap B(z, r) .
$$

By Lemma 4.6, we have

$$
\begin{aligned}
I_{2}(x) \leq & C \sum_{j=1}^{j_{0}} \int_{B\left(z, \vartheta c^{j} r\right) \backslash B\left(z, \vartheta c^{j-1} r\right)} \frac{d(z, y)^{\gamma}}{\mu(B(z, c d(z, y)))} f(y) d \mu(y) \\
\leq & C \sum_{j=1}^{j_{0}}\left(\vartheta c^{j} r\right)^{\gamma} \frac{1}{\mu\left(B\left(z, \vartheta c^{j} r\right)\right)} \int_{X \cap B\left(z, \vartheta c^{j} r\right)} f(y) d \mu(y) \\
\leq & C\left(\sum_{j=1}^{j_{0}-1}\left(\vartheta c^{j} r\right)^{\gamma} \Phi^{-1}\left(x, \kappa\left(x, \vartheta c^{j} r\right)^{-1}\right) \eta\left(\left(\log \left(e+1 /\left(\vartheta c^{j} r\right)\right)\right)^{-1}\right)^{-1}\right. \\
& \left.+d_{X}^{\gamma} \Phi^{-1}\left(x, \kappa\left(x, d_{X}\right)^{-1}\right) \eta\left(\left(\log \left(e+1 / d_{X}\right)\right)^{-1}\right)^{-1}\right),
\end{aligned}
$$

where we assume that $\sum_{j=1}^{0} a_{j}=0$ for $a_{j} \in \mathbf{R}$. As in the proof of Lemma 4.8, we obtain

$$
\begin{aligned}
I_{2}(x) \leq & \frac{C}{\log c}\left(\sum_{j=1}^{j_{0}-1} \int_{\vartheta_{c^{j-1} r}}^{\vartheta c^{j} r} \rho^{\gamma} \Phi^{-1}\left(x, \kappa(x, \rho)^{-1}\right) \eta\left((\log (e+1 / \rho))^{-1}\right)^{-1} \frac{d \rho}{\rho}\right. \\
& \left.+\int_{d_{X} / 2}^{d_{X}} \rho^{\gamma} \Phi^{-1}\left(x, \kappa(x, \rho)^{-1}\right) \eta\left((\log (e+1 / \rho))^{-1}\right)^{-1} \frac{d \rho}{\rho}\right) \\
\leq & C \int_{r}^{d_{X}} \rho^{\gamma} \Phi^{-1}\left(z, \kappa(z, \rho)^{-1}\right) \eta\left((\log (e+1 / \rho))^{-1}\right)^{-1} \frac{d \rho}{\rho} \\
\leq & \frac{C}{\log c} \frac{1}{\lambda_{\gamma}(z, r)}
\end{aligned}
$$

for all $x \in X \cap B(z, r)$. Hence,

$$
\int_{X \cap B(z, r)} I_{2}(x) d \mu(x) \leq \frac{C}{\log c} \frac{\mu(B(z, r))}{\lambda_{\gamma}(z, r)} \leq \frac{C}{\log c} \frac{\mu(B(z, \vartheta r))}{\lambda_{\gamma}(z, r)} .
$$

Thus, this lemma is proved.

\section{Trudinger's inequality for grand Musielak-Orlicz-Morrey spaces}

In this section, we deal with the case in which $\Gamma(x, t)$ satisfies the uniform log-type condition: 
$\left(\Gamma_{\log }\right)$ there exists a constant $c_{\Gamma}>0$ such that

$$
\Gamma\left(x, t^{2}\right) \leq c_{\Gamma} \Gamma(x, t)
$$

for all $x \in X$ and $t \geq 1$.

By $\left(\Gamma_{\log }\right)$, together with Lemma 4.7 , we see that $\Gamma(x, t)$ satisfies the uniform doubling condition in $t$.

LEMMA 5.1 (CF. [14, LEMMA 4.2])

Suppose $\Gamma(x, t)$ satisfies $\left(\Gamma_{\log }\right)$. For every $a>1$, there exists $b>0$ such that $\Gamma(x, a t) \leq b \Gamma(x, t)$ for all $x \in X$ and $t>0$.

\section{THEOREM 5.2}

Assume that $\Phi(x, t)$ satisfies $(\Phi 5)$ and $\Gamma(x, t)$ satisfies $\left(\Gamma_{\log }\right)$. For each $x \in X$, let $\gamma(x)=\sup _{s>0} \Gamma(x, s)$. Suppose $\Psi(x, t): X \times[0, \infty) \rightarrow[0, \infty]$ satisfies the following conditions:

$(\Psi 1) \Psi(\cdot, t)$ is measurable on $X$ for each $t \in[0, \infty)$ and $\Psi(x, \cdot)$ is continuous on $[0, \infty)$ for each $x \in X$;

( $\Psi 2)$ there is a constant $A_{1}^{\prime} \geq 1$ such that $\Psi(x, t) \leq \Psi\left(x, A_{1}^{\prime} s\right)$ for all $x \in X$ whenever $0<t<s$;

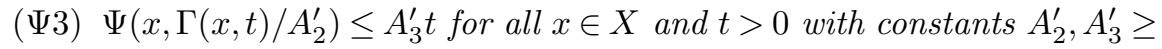
1 independent of $x$.

Let $\tau>2$ and $\vartheta>1$ be such that $\tau>(\vartheta+1) /(\vartheta-1)$. Then, for $0<\gamma<\alpha$, there exists a constant $C^{*}>0$ such that $I_{\alpha, \tau} f(x) / C^{*}<\gamma(x)$ for almost every $x \in X$ and

$$
\frac{\lambda_{\gamma}(z, r)}{\mu(B(z, \vartheta r))} \int_{X \cap B(z, r)} \Psi\left(x, \frac{I_{\alpha, \tau} f(x)}{C^{*}}\right) d \mu(x) \leq 1
$$

for all $z \in X, 0<r<d_{X}$, and nonnegative $f \in \widetilde{L}_{\eta, \xi}^{\Phi, \kappa}(X)$ with $\|f\|_{\Phi, \kappa ; \eta, \xi ; X} \leq 1$.

\section{Proof}

Let $f \geq 0$ and $\|f\|_{\Phi, \kappa ; \eta, \xi ; X} \leq 1$. Fix $x \in X$. For $0<\delta \leq d_{X} / 2$, Lemma 4.8 implies

$$
\begin{aligned}
I_{\alpha, \tau} f(x) & \leq \int_{X \cap B(x, \delta)} \frac{d(x, y)^{\alpha} f(y)}{\mu(B(x, \tau d(x, y)))} d \mu(y)+C \Gamma\left(x, \frac{1}{\delta}\right) \\
& =\int_{X \cap B(x, \delta)} d(x, y)^{\alpha-\gamma} \frac{d(x, y)^{\gamma} f(y)}{\mu(B(x, \tau d(x, y)))} d \mu(y)+C \Gamma\left(x, \frac{1}{\delta}\right) \\
& \leq C\left\{\delta^{\alpha-\gamma} I_{\gamma, \tau} f(x)+\Gamma\left(x, \frac{1}{\delta}\right)\right\}
\end{aligned}
$$

with constants $C>0$ independent of $x$.

If $I_{\gamma, \tau} f(x) \leq 2 / d_{X}$, then we take $\delta=d_{X} / 2$. Then, by Lemma 4.7

$$
I_{\alpha, \tau} f(x) \leq C \Gamma\left(x, \frac{2}{d_{X}}\right) .
$$


By Lemma 5.1, there exists $C_{1}^{*}>0$ independent of $x$ such that

$$
I_{\alpha, \tau} f(x) \leq C_{1}^{*} \Gamma\left(x, \frac{1}{2 A_{3}^{\prime}}\right) \quad \text { if } I_{\gamma, \tau} f(x) \leq 2 / d_{X} .
$$

Next, suppose $2 / d_{X}<I_{\gamma, \tau} f(x)<\infty$. Let $m=\sup _{s \geq 2 / d_{X}, x \in X} \Gamma(x, s) / s$. By $\left(\Gamma_{\log }\right), m<\infty$. Define $\delta$ by

$$
\delta^{\alpha-\gamma}=\frac{\left(d_{X} / 2\right)^{\alpha-\gamma}}{m} \Gamma\left(x, I_{\gamma, \tau} f(x)\right)\left(I_{\gamma, \tau} f(x)\right)^{-1} .
$$

Since $\Gamma\left(x, I_{\gamma, \tau} f(x)\right)\left(I_{\gamma, \tau} f(x)\right)^{-1} \leq m, 0<\delta \leq d_{X} / 2$. Then by Lemma 4.7

$$
\begin{aligned}
\frac{1}{\delta} & \leq C \Gamma\left(x, I_{\gamma, \tau} f(x)\right)^{-1 /(\alpha-\gamma)}\left(I_{\gamma, \tau} f(x)\right)^{1 /(\alpha-\gamma)} \\
& \leq C \Gamma\left(x, 2 / d_{X}\right)^{-1 /(\alpha-\gamma)}\left(I_{\gamma, \tau} f(x)\right)^{1 /(\alpha-\gamma)} \\
& \leq C\left(I_{\gamma, \tau} f(x)\right)^{1 /(\alpha-\gamma)} .
\end{aligned}
$$

Hence, using $\left(\Gamma_{\log }\right)$ and Lemma 5.1, we obtain

$$
\Gamma\left(x, \frac{1}{\delta}\right) \leq \Gamma\left(x, C\left(I_{\gamma, \tau} f(x)\right)^{1 /(\alpha-\gamma)}\right) \leq C \Gamma\left(x, I_{\gamma, \tau} f(x)\right) .
$$

By Lemma 5.1 again, we see that there exists a constant $C_{2}^{*}>0$ independent of $x$ such that

$$
I_{\alpha, \tau} f(x) \leq C_{2}^{*} \Gamma\left(x, \frac{1}{2 C_{I, \gamma} A_{3}^{\prime}} I_{\gamma, \tau} f(x)\right) \quad \text { if } 2 / d_{X}<I_{\gamma, \tau} f(x)<\infty,
$$

where $C_{I, \gamma}$ is the constant given in Lemma 4.9 .

Now, let $C^{*}=A_{1}^{\prime} A_{2}^{\prime} \max \left(C_{1}^{*}, C_{2}^{*}\right)$. Then, by (5.1) and (5.2),

$$
\frac{I_{\alpha, \tau} f(x)}{C^{*}} \leq \frac{1}{A_{1}^{\prime} A_{2}^{\prime}} \max \left\{\Gamma\left(x, \frac{1}{2 A_{3}^{\prime}}\right), \Gamma\left(x, \frac{1}{2 C_{I, \gamma} A_{3}^{\prime}} I_{\gamma, \tau} f(x)\right)\right\}
$$

whenever $I_{\gamma, \tau} f(x)<\infty$. Since $I_{\gamma, \tau} f(x)<\infty$ for almost every $x \in X$ by Lemma $4.9, I_{\alpha, \tau} f(x) / C^{*}<\gamma(x)$ for almost every $x \in X$, and by $(\Psi 2)$ and $(\Psi 3)$, we have

$$
\begin{aligned}
& \Psi\left(x, \frac{I_{\alpha, \tau} f(x)}{C^{*}}\right) \\
& \quad \leq \max \left\{\Psi\left(x, \Gamma\left(x, \frac{1}{2 A_{3}^{\prime}}\right) / A_{2}^{\prime}\right), \Psi\left(x, \Gamma\left(x, \frac{1}{2 C_{I, \gamma} A_{3}^{\prime}} I_{\gamma, \tau} f(x)\right) / A_{2}^{\prime}\right)\right\} \\
& \quad \leq \frac{1}{2}+\frac{1}{2 C_{I, \gamma}} I_{\gamma, \tau} f(x)
\end{aligned}
$$

for almost every $x \in X$. Thus, noting that $\lambda_{\gamma}(z, r) \leq 1$ and using Lemma 4.9, we have

$$
\begin{aligned}
& \frac{\lambda_{\gamma}(z, r)}{\mu(B(z, \vartheta r))} \int_{X \cap B(z, r)} \Psi\left(x, \frac{I_{\alpha, \tau} f(x)}{C^{*}}\right) d \mu(x) \\
& \quad \leq \frac{1}{2} \lambda_{\gamma}(z, r)+\frac{1}{2 C_{I, \gamma}} \frac{\lambda_{\gamma}(z, r)}{\mu(B(z, \vartheta r))} \int_{X \cap B(z, r)} I_{\gamma, \tau} f(x) d \mu(x)
\end{aligned}
$$




$$
\begin{aligned}
& \leq \frac{1}{2}+\frac{1}{2} \\
& =1
\end{aligned}
$$

for all $z \in X$ and $0<r<d_{X}$.

\section{REMARK 5.3}

If $\Gamma(x, s)$ is bounded, that is,

$$
\sup _{x \in X} \int_{0}^{d_{X}} \rho^{\alpha} \Phi^{-1}\left(x, \kappa(x, \rho)^{-1}\right) \eta\left((\log (e+1 / \rho))^{-1}\right)^{-1} d \rho<\infty,
$$

then by Lemma 4.8 we see that $I_{\alpha, \tau}|f|$ is bounded for every $f \in \widetilde{L}_{\eta, \xi}^{\Phi, \kappa}(X)$.

\section{REMARK 5.4}

We cannot take $\gamma=\alpha$ in Theorem 5.2. For details, see [17, Remark 2.8].

As in the proof of [14, Corollary 4.6], we obtain the following corollary by applying Theorem 5.2 to the special $\Phi$ and $\kappa$ given in Examples 2.1 and 2.2.

\section{COROLLARY 5.5}

Let $\kappa$ be as in Example 2.2, and let $p(x)$ and $q(x)=q_{1}(x)$ be as in Example 2.1. Let $\tau>2$ and $\vartheta>1$ be such that $\tau>(\vartheta+1) /(\vartheta-1)$. Set $\eta(t)=t^{\theta}$ for $\theta>0$ and $\Phi(x, t)=t^{p(x)}(\log (e+t))^{q(x)}$.

Assume that

$$
\alpha-\nu(x) / p(x)=0 \quad \text { for all } x \in X \text {. }
$$

(1) Suppose

$$
\inf _{x \in X}(-q(x) / p(x)-\beta(x) / p(x)+\theta+1)>0 .
$$

Then for $0<\gamma<\alpha$ there exist constants $C^{*}>0$ and $C^{* *}>0$ such that

$$
\frac{r^{\nu(z) / p(z)-\gamma}}{\mu(B(z, \vartheta r))} \int_{B(z, r) \cap X} \exp \left(\left(\frac{I_{\alpha, \tau} f(x)}{C^{*}}\right)^{p(x) /(p(x)+\theta p(x)-\beta(x)-q(x))}\right) d \mu(x) \leq C^{* *}
$$

for all $z \in X, 0<r \leq d_{X}$, and nonnegative $f \in \widetilde{L}_{\eta, \xi}^{\Phi, \kappa}(X)$ with $\|f\|_{\Phi, \kappa ; \eta, \xi ; X} \leq 1$.

(2) If

$$
\sup _{x \in X}(-q(x) / p(x)-\beta(x) / p(x)+\theta+1) \leq 0,
$$

then for $0<\gamma<\alpha$ there exist constants $C^{*}>0$ and $C^{* *}>0$ such that

$$
\frac{r^{\nu(z) / p(z)-\gamma}}{\mu(B(z, \vartheta r))} \int_{B(z, r) \cap X} \exp \left(\exp \left(\frac{I_{\alpha, \tau} f(x)}{C^{*}}\right)\right) d \mu(x) \leq C^{* *}
$$

for all $z \in X, 0<r<d_{X}$, and nonnegative $f \in \widetilde{L}_{\eta, \xi}^{\Phi, \kappa}(X)$ with $\|f\|_{\Phi, \kappa ; \eta, \xi ; X} \leq 1$. 


\section{Continuity for grand Musielak-Orlicz-Morrey spaces}

In this section, we discuss the continuity of Riesz potentials $I_{\alpha, \tau} f$ of functions in grand Musielak-Orlicz-Morrey spaces under the condition that there are constants $\theta>0, \iota>1$, and $C_{0}>0$ such that

$$
\left|\frac{d(x, y)^{\alpha}}{\mu(B(x, \tau d(x, y)))}-\frac{d(z, y)^{\alpha}}{\mu(B(z, \tau d(z, y)))}\right| \leq C_{0}\left(\frac{d(x, z)}{d(x, y)}\right)^{\theta} \frac{d(x, y)^{\alpha}}{\mu(B(x, \iota d(x, y)))}
$$

whenever $d(x, z) \leq d(x, y) / 2$.

We consider the functions

$$
\omega(x, r)=\int_{0}^{r} \rho^{\alpha} \Phi^{-1}\left(x, \kappa(x, \rho)^{-1}\right) \eta\left((\log (e+1 / \rho))^{-1}\right)^{-1} \frac{d \rho}{\rho}
$$

and

$$
\omega_{\theta}(x, r)=r^{\theta} \int_{r}^{d_{X}} \rho^{\alpha-\theta} \Phi^{-1}\left(x, \kappa(x, \rho)^{-1}\right) \eta\left((\log (e+1 / \rho))^{-1}\right)^{-1} \frac{d \rho}{\rho}
$$

for $\theta>0$ and $0<r \leq d_{X}$.

LEMMA 6.1 (CF. [14, LEMMA 5.1])

Let $E \subset X$. If $\omega(x, r) \rightarrow 0$ as $r \rightarrow 0+$ uniformly in $x \in E$, then $\omega_{\theta}(x, r) \rightarrow 0$ as $r \rightarrow 0+$ uniformly in $x \in E$.

\section{LEMMA 6.2 (CF. [14, LEMMA 5.2])}

There exists a constant $C>0$ such that

$$
\omega(x, 2 r) \leq C \omega(x, r)
$$

for all $x \in X$ and $0<r \leq d_{X} / 2$.

\section{THEOREM 6.3}

Assume that $\Phi(x, t)$ satisfies $(\Phi 5)$. Let $\tau>1$. Then there exists a constant $C>0$ such that

$$
\left|I_{\alpha, \tau} f(x)-I_{\alpha, \tau} f(z)\right| \leq C\left\{\omega(x, d(x, z))+\omega(z, d(x, z))+\omega_{\theta}(x, d(x, z))\right\}
$$

for all $x, z \in X$ with $d(x, z) \leq d_{X} / 4$ and nonnegative $f \in \widetilde{L}_{\eta, \xi}^{\Phi, \kappa}(X)$ with $\|f\|_{\Phi, \kappa ; \eta, \xi ; X} \leq 1$.

Before giving a proof of Theorem 6.3, we prepare two more lemmas.

LEMMA 6.4

Assume that $\Phi(x, t)$ satisfies $(\Phi 5)$. Let $\tau>1$, and let $f$ be a nonnegative function on $X$ such that $\|f\|_{\Phi, \kappa ; \eta, \xi ; X} \leq 1$. Then there exists a constant $C>0$ such that

$$
\int_{X \cap B(x, \delta)} \frac{d(x, y)^{\alpha} f(y)}{\mu(B(x, \tau d(x, y)))} d \mu(y) \leq C \omega(x, \delta)
$$

for all $x \in X$ and $0<\delta \leq d_{X}$. 


\section{Proof}

Let $f$ be a nonnegative function on $X$ with $\|f\|_{\Phi, \kappa ; \eta, \xi ; X} \leq 1$. As usual we start by decomposing $B(x, \delta)$ dyadically:

$$
\begin{aligned}
& \int_{X \cap B(x, \delta)} \frac{d(x, y)^{\alpha} f(y)}{\mu(B(x, \tau d(x, y)))} d \mu(y) \\
& \quad=\sum_{j=1}^{\infty} \int_{X \cap\left(B\left(x, \tau^{-j+1} \delta\right) \backslash B\left(x, \tau^{-j} \delta\right)\right)} \frac{d(x, y)^{\alpha} f(y)}{\mu(B(x, \tau d(x, y)))} d \mu(y) \\
& \quad \leq \sum_{j=1}^{\infty}\left(\tau^{-j+1} \delta\right)^{\alpha} \frac{1}{\mu\left(B\left(x, \tau^{-j+1} \delta\right)\right)} \int_{B\left(x, \tau^{-j+1} \delta\right)} f(y) d \mu(y) .
\end{aligned}
$$

By Lemma 4.6, we have

$$
\begin{aligned}
& \int_{X \cap B(x, \delta)} \frac{d(x, y)^{\alpha} f(y)}{\mu(B(x, \tau d(x, y)))} d \mu(y) \\
& \quad \leq C \sum_{j=1}^{\infty}\left(\tau^{-j+1} \delta\right)^{\alpha} \Phi^{-1}\left(x, \kappa\left(x, \tau^{-j+1} \delta\right)^{-1}\right) \eta\left(\left(\log \left(e+1 /\left(\tau^{-j+1} \delta\right)\right)\right)^{-1}\right)^{-1} \\
& \quad \leq \frac{C}{\log \tau} \int_{0}^{\delta} \rho^{\alpha} \Phi^{-1}\left(x, \kappa(x, \rho)^{-1}\right) \eta\left((\log (e+1 / \rho))^{-1}\right)^{-1} \frac{d \rho}{\rho} \\
& \quad=C \omega(x, \delta)
\end{aligned}
$$

The following lemma can be proved in the same manner as Lemma 4.8.

\section{LEMMA 6.5}

Assume that $\Phi(x, t)$ satisfies $(\Phi 5)$. Let $\theta \in \mathbf{R}$, and let $\tau>1$. Let $f$ be a nonnegative function on $X$ such that $\|f\|_{\Phi, \kappa ; \eta, \xi ; X} \leq 1$. Then there exists a constant $C>0$ such that

$$
\int_{X \backslash B(x, \delta)} \frac{d(x, y)^{\alpha-\theta} f(y)}{\mu(B(x, \tau d(x, y)))} d \mu(y) \leq C \delta^{-\theta} \omega_{\theta}(x, \delta)
$$

for all $x \in X$ and $0<\delta \leq d_{X} / 2$.

\section{Proof of Theorem 6.3}

Let $f$ be a nonnegative function on $X$ with $\|f\|_{\Phi, \kappa ; \eta, \xi ; X} \leq 1$, and let $x, z \in X$ with $d(x, z) \leq d_{X} / 4$. Write

$$
\begin{aligned}
& I_{\alpha, \tau} f(x)-I_{\alpha, \tau} f(z) \\
& =\int_{X \cap B(x, 2 d(x, z))} \frac{d(x, y)^{\alpha} f(y)}{\mu(B(x, \tau d(x, y)))} d \mu(y) \\
& \quad-\int_{X \cap B(x, 2 d(x, z))} \frac{d(z, y)^{\alpha} f(y)}{\mu(B(z, \tau d(z, y)))} d \mu(y) \\
& \quad+\int_{X \backslash B(x, 2 d(x, z))}\left(\frac{d(x, y)^{\alpha}}{\mu(B(x, \tau d(x, y)))}-\frac{d(z, y)^{\alpha}}{\mu(B(z, \tau d(z, y)))}\right) f(y) d \mu(y) .
\end{aligned}
$$


Using Lemmas 6.2 and 6.4, we have

$$
\int_{X \cap B(x, 2 d(x, z))} \frac{d(x, y)^{\alpha} f(y)}{\mu(B(x, \tau d(x, y)))} d \mu(y) \leq C \omega(x, 2 d(x, z)) \leq C \omega(x, d(x, z))
$$

and

$$
\begin{aligned}
\int_{X \cap B(x, 2 d(x, z))} \frac{d(z, y)^{\alpha} f(y)}{\mu(B(z, \tau d(z, y)))} d \mu(y) & \leq \int_{X \cap B(z, 3 d(x, z))} \frac{d(z, y)^{\alpha} f(y)}{\mu(B(z, \tau d(z, y)))} d \mu(y) \\
& \leq C \omega(z, 3 d(x, z)) \leq C \omega(z, d(x, z)) .
\end{aligned}
$$

On the other hand, by (6.1) and Lemma 6.5, we have

$$
\begin{aligned}
& \int_{X \backslash B(x, 2 d(x, z))}\left|\frac{d(x, y)^{\alpha}}{\mu(B(x, \tau d(x, y)))}-\frac{d(z, y)^{\alpha}}{\mu(B(z, \tau d(z, y)))}\right| f(y) d \mu(y) \\
& \leq C d(x, z)^{\theta} \int_{X \backslash B(x, 2 d(x, z))} \frac{d(x, y)^{\alpha-\theta} f(y)}{\mu(B(x, \iota d(x, y)))} d \mu(y) \\
& \leq C \omega_{\theta}(x, 2 d(x, z)) \leq C \omega_{\theta}(x, d(x, z)) .
\end{aligned}
$$

Then we have the conclusion.

In view of Lemma 6.1, we obtain the following corollary.

\section{COROLLARY 6.6}

Assume that $\Phi(x, t)$ satisfies $(\Phi 5)$. Let $\tau>1$.

(a) Let $x_{0} \in X$, and suppose $\omega(x, r) \rightarrow 0$ as $r \rightarrow 0+$ uniformly in $x \in X \cap$ $B\left(x_{0}, \delta\right)$ for some $\delta>0$. Then $I_{\alpha, \tau} f$ is continuous at $x_{0}$ for every $f \in \widetilde{L}_{\eta, \xi}^{\Phi, \kappa}(X)$.

(b) Suppose $\omega(x, r) \rightarrow 0$ as $r \rightarrow 0+$ uniformly in $x \in X$. Then $I_{\alpha, \tau} f$ is uniformly continuous on $X$ for every $f \in \widetilde{L}_{\eta, \xi}^{\Phi, \kappa}(X)$.

\section{References}

[1] D. R. Adams, A note on Riesz potentials, Duke Math. J. 42 (1975), 765-778. MR 0458158.

[2] D. R. Adams and L. I. Hedberg, Function Spaces and Potential Theory, Grundlehren Math. Wiss. 314, Springer, Berlin, 1996. MR 1411441. DOI 10.1007/978-3-662-03282-4.

[3] C. Capone, M. R. Formica, and R. Giova, Grand Lebesgue spaces with respect to measurable functions, Nonlinear Anal. 85 (2013), 125-131. MR 3040353.

DOI 10.1016/j.na.2013.02.021.

[4] F. Farroni and R. Giova, The distance to $L^{\infty}$ in the grand Orlicz spaces, J. Funct. Spaces Appl. 2013, no. 658527. MR 3102761.

[5] A. Fiorenza and C. Sbordone, Existence and uniqueness results for solutions of nonlinear equations with right hand side in $L^{1}$, Studia Math. 127 (1998), 223-231. MR 1489454. 
[6] T. Futamura, Y. Mizuta, and T. Ohno, "Sobolev's theorem for Riesz potentials of functions in grand Morrey spaces of variable exponent" in Banach and Function Spaces, IV (ISBFS 2012), Yokohama Publ., Yokohama, 2014, 353-365. MR 3289785.

[7] L. Greco, T. Iwaniec, and C. Sbordone, Inverting the p-harmonic operator, Manuscripta Math. 92 (1997), 249-258. MR 1428651.

DOI 10.1007/BF02678192.

[8] P. Hajłasz and P. Koskela, Sobolev met Poincaré, Mem. Amer. Math. Soc. 145 (2000), no. 688. MR 1683160. DOI 10.1090/memo/0688.

[9] T. Iwaniec and C. Sbordone, On the integrability of the Jacobian under minimal hypotheses, Arch. Rational Mech. Anal. 119 (1992), 129-143. MR 1176362. DOI 10.1007/BF00375119.

[10] , Riesz transforms and elliptic PDEs with VMO coefficients, J. Anal. Math. 74 (1998), 183-212. MR 1631658. DOI 10.1007/BF02819450.

[11] V. Kokilashvili, A. Meskhi, and H. Rafeiro, Riesz type potential operators in generalized grand Morrey spaces, Georgian Math. J. 20 (2013), 43-64. MR 3037076. DOI 10.1515/gmj-2013-0009.

[12] P. Koskela and X. Zhong, Minimal assumptions for the integrability of the Jacobian, Ricerche Mat. 51 (2002), 297-311. MR 2030546.

[13] F.-Y. Maeda, Y. Mizuta, T. Ohno, and T. Shimomura, Boundedness of maximal operators and Sobolev's inequality on Musielak-Orlicz-Morrey spaces, Bull. Sci. Math. 137 (2013), 76-96. MR 3007101. DOI 10.1016/j.bulsci.2012.03.008.

[14] Trudinger's inequality and continuity of potentials on Musielak-Orlicz-Morrey spaces, Potential Anal. 38 (2013), 515-535. MR 3015362. DOI 10.1007/s11118-012-9284-y.

[15] S Sobolev and Trudinger type inequalities on grand Musielak-Orlicz-Morrey spaces, Ann. Acad. Sci. Fenn. Math. 40 (2015), 403-426. MR 3329151. DOI 10.5186/aasfm.2015.4027.

[16] A. Meskhi, Maximal functions, potentials and singular integrals in grand Morrey spaces, Complex Var. Elliptic Equ. 56 (2011), 1003-1019. MR 2838234. DOI 10.1080/17476933.2010.534793.

[17] Y. Mizuta, E. Nakai, T. Ohno, and T. Shimomura, An elementary proof of Sobolev embeddings for Riesz potentials of functions in Morrey spaces $L^{1, \nu, \beta}(G)$, Hiroshima Math. J. 38 (2008), 425-436. MR 2477751.

[18] B Boundedness of fractional integral operators on Morrey spaces and Sobolev embeddings for generalized Riesz potentials, J. Math. Soc. Japan 62 (2010), 707-744. MR 2648060.

[19] , Riesz potentials and Sobolev embeddings on Morrey spaces of variable exponents, Complex Var. Elliptic Equ. 56 (2011), 671-695. MR 2832209. DOI 10.1080/17476933.2010.504837.

[20] Y. Mizuta and T. Shimomura, Sobolev embeddings for Riesz potentials of functions in Morrey spaces of variable exponent, J. Math. Soc. Japan 60 (2008), 583-602. MR 2421989. 
[21] Continuity properties for Riesz potentials of functions in Morrey spaces of variable exponent, Math. Inequal. Appl. 13 (2010), 99-122. MR 2648234. DOI 10.7153/mia-13-08.

[22] Y. Mizuta, T. Shimomura, and T. Sobukawa, Sobolev's inequality for Riesz potentials of functions in non-doubling Morrey spaces, Osaka J. Math. 46 (2009), 255-271. MR 2531149.

[23] E. Nakai, "Generalized fractional integrals on Orlicz-Morrey spaces" in Banach and Function Spaces, Yokohama Publ., Yokohama, 2004, 323-333. MR 2146936.

[24] T. Ohno and T. Shimomura, Trudinger's inequality for Riesz potentials of functions in Musielak-Orlicz spaces, Bull. Sci. Math. 138 (2014), 225-235. MR 3175020. DOI 10.1016/j.bulsci.2013.05.007.

[25] H. Rafeiro, "A note on boundedness of operators in grand grand Morrey spaces" in Advances in Harmonic Analysis and Operator Theory, Oper. Theory Adv. Appl. 229, Birkhäuser/Springer Basel AG, Basel, 2013, 349-356.

MR 3060423. DOI 10.1007/978-3-0348-0516-2_19.

[26] Y. Sawano and T. Shimomura, Sobolev embeddings for Riesz potentials of functions in non-doubling Morrey spaces of variable exponents, Collect. Math. 64 (2013), 313-350. MR 3084400. DOI 10.1007/s13348-013-0082-7.

[27] Sobolev embeddings for Riesz potentials of functions in Musielak-Orlicz-Morrey spaces over non-doubling measure spaces, Integral Transforms Spec. Funct. 25 (2014), 976-991. MR 3267751. DOI 10.1080/10652469.2014.955099.

[28] C. Sbordone, Grand Sobolev spaces and their applications to variational problems, Matematiche (Catania) 51 (1996), 335-347. MR 1488076.

Ohno: Faculty of Education and Welfare Science, Oita University, Dannoharu, Oita-city, Japan; t-ohno@oita-u.ac.jp

Shimomura: Department of Mathematics, Graduate School of Education, Hiroshima University, Higashi-Hiroshima, Japan; tshimo@hiroshima-u.ac.jp 